\title{
Designing for Improving Verbal Patient Transfer
}

\author{
Abdullah Al Mahmud, Max Eichenbrenner, and Omar Mubin \\ Department of Industrial Design, \\ Eindhoven University of Technology, \\ P.O. Box 513, 5600 MB Eindhoven, The Netherlands \\ a.al-mahmud@tue.nl, o.mubin@tue.nl
}

\begin{abstract}
The current verbal patient transfer between nurses and paramedics lacks communication, which leads to loss of information. During this operation, handwritten protocols are used to document the patient's personal data and the treatment given at several intervals. In order to encourage teamwork between the two parties (i.e. nurses and paramedics), the challenge is to present the patient information in a more accessible and feasible way. In this paper, we present the design and evaluation of an interactive and tangible presentation medium that aims to improve the verbal patient transfer by mediated communication. Our result shows that the concept would improve the current verbal patient transfer as evaluated by paramedics and nurses.
\end{abstract}

\section{Introduction}

In the pre-hospital phase of an emergency, medical technician and paramedics provide patients with treatment, care and qualified transport to the hospital. During this operation, handwritten protocols are used to document the patient's personal data, the anamnesis and the treatment given at several time intervals. The verbal patient transfer (VPT) is the crucial joint between the pre-hospital and hospital treatment of the patient where information in condensed form has to be presented and understood. It has been observed that the traditional form of VPT in hospitals leads to miscommunication and information loss between the emergency medical technician-paramedics, short paramedics, and nurses [3], [4]. It is stated that the majority of the VPT is only 'partly' performed according to a standard guidelines [2]. In perspective of the nurses, it is stated that most VPT would provide only 'partly' useful information and it is better to gather information on your own instead of listening to the paramedics. Currently used operation protocols would be designed for the information need of a doctor, whereas in hospital most VPT are performed between nurses and paramedics. Even sometimes verbal handover is done without filling the protocol [6]. Further, it is suggested that the communication between the two parties needs to be drastically intensified, to improve the VPT [1]. The goal for this work therefore is to design an interactive and tangible presentation medium to improve the verbal patient transfer by mediated communication. More precisely, the aim is to help improving the verbal patient transfer (VPT), to enhance the quality of the patient treatment and to prevent miscommunication and information loss that may lead to death of patients in worst cases. 


\section{Design Approach}

It was necessary to understand the current workflow and verbal patient transfer (VPT). Therefore, two different research approaches have been followed namely, the Activity-Centered Design (ACD) and the User-Centered Design (UCD) approach [5]. Information was gathered through semi-structured interviews and observations in the work environment A total seven paramedics, seven nurses and two doctors from five hospitals and four fire departments were interviewed in several iterations to gather insights about the workflow and the social coherence of information during patient transfers.

Verbal Patient Transfer (VPT). The quality of the VPT is highly dependant on the presenter and the receiver type. In case a doctor is present, it was stated that the VPT was generally better performed by the paramedics. The interviewed paramedics had the belief that VPT performed with the nurses was problematic. Main sources of miscommunication and information loss could be defined as: the mental state of the presenter (i.e. nervous, less conscious and unmotivated), the missing structure (i.e. coherent story line) and missing abstraction (i.e. highlight level of importance). The paramedics stated confusion, caused by similar operations and forgetting details as the major sources of miscommunication and information loss. Concerning the interaction, the paramedics stated that they would miss the nurse's feedback, whether the information was understood.

Protocol. An interview with an experienced attendant revealed that the operation protocols are commonly thrown in the bin without putting an effort in evaluating them. Interviewed nurses stated "the changing status of the patient and the tendency of higher measurements during the transport" as the reason for neglecting the protocols. In summary, the nurses did not perceive the protocol as their responsibility. One interviewee mentioned she experienced frustration with the poorly written protocols. Generally, it can be stated that the current handwritten protocols find no acceptance by the nurses and thus are not suitable as supporting element of the VPT.

Concept Design and Implementation. Based on the above results, consultation with nurses and paramedics a concept of intelligent table named 'DACCORD' was developed. It uses a so-called data terminal, a laptop with a touch screen and UMTS/VPN connection, to send the patient information in the form of an .xml document from the ambulance directly to the hospital. The DACCORD concept (Fig. 1) transfers this .xml document into an interactive interface. Two different access points located opposite each other are designed to fit the individual tasks of the communication partners. Both sides are equipped with a separate screen showing the same content. The presenter's (paramedic) side uses a slider for navigating through the interface. The receiver's (nurse or doctor) side is equipped with a notepad, to add information and an accept buzzer. The accept buzzer allows the information receiver to give feedback whether he/she understands and agrees. An additional LED light on each side of the table, called side leafs, are depicting information of the level of emergence by color. The two table halves were positioned in a 15-degree angle, which allows good visibility of the screen in front and slight visibility of the other screen. Together with an expert from the fire department and the hospital, two cases were defined which were representative for the whole range of operations. 

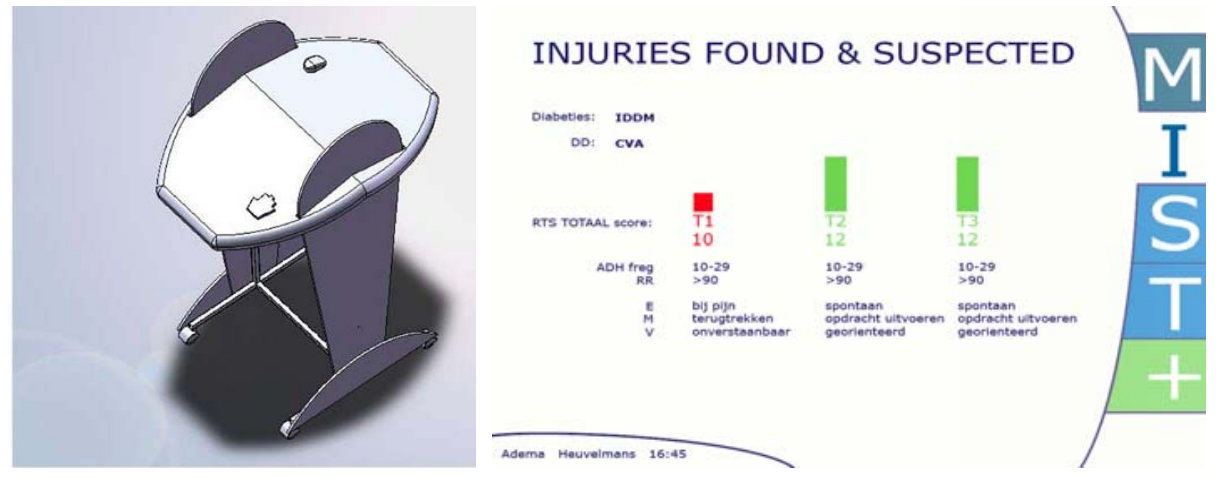

Fig. 1. Implementation: table (left), interface layout (right)

It was decided to define an emergent case, a poly-trauma, and a less emergent case in the form of a diabetes patient. To create the interface, the currently used handwritten protocol and the digital protocol were analyzed. The digital protocol was completed by an expert from the fire department according to the two cases. Furthermore, the official guideline in the Netherlands, called the 'MIST' (i.e. measurements, injuries, signs and treatment) schema, which describes content of the VPT was studied. Based on the documents, first versions of the interface were sketched. Depending on the sketches a digital version of the interface was created. To create the content, relevant information was filtered from the .xml document of the completed digital protocol and the handwritten protocol. This information was then sorted into the basic structure of the official 'MIST scheme'. The Revised Trauma Score (RTS), composed of a combination of results from Glasgow Coma Scale, Systolic Blood Pressure, and respiratory rate was transferred into a graph. This graph (Fig. 1) illustrates in form of bars by three different sizes and colors the condition of the patient over time.

\section{Evaluation}

In total two tests were performed with four users. For each user test one nurse or attendant and one paramedic participated. The user evaluation aimed for questions concerning usability and user experience that could possibly lead to improvements of the design. The user test was divided into two steps. Firstly, the system was introduced and time for one individual 'practice round' was given. Secondly, the participants got a real case and were asked to perform a VPT using the system. To make sure, the participants use the full range of functions, they received a task list beforehand. The session closed with an interview.

Overall, the DACCORD concept was understood by all of the medical staff. Based on the short instructions, the participants were able to perform the 'practice round' individually. The instruction including the 'practice round' of the system took 10 minutes and should be a part of the real deployment of the DACCORD table. The system helped the paramedics to present the information in a more structured manner. Statements such as "clear interface, you cannot miss it. It goes step by step", support this conclusion. The system aided the nurses in the VPT process. This can be 
reasoned by observing the following qualitative remarks: "I can see the told information", "No more information is presented than I need", "I do not have to search" and "I have control of accepting" (in reference to the accept button). The system demands to process auditory and visual information at the same time. This was considered as 'easy' by all participants. As an improvement, participants suggested a clear distinction between the two access points. Furthermore, the nurses and paramedics suggested being in control of the uploading of the patient data instead of the server. It should be noted that the total number of four users is not sufficient but we were restricted by the availability of the user group in a real setting. The test could not include a patient, who could cause interruptions and unexpected behavior during a natural VPT process. Furthermore, the test worked with an already filled out protocol. In future, we will compare previous systems with the DACCORD prototype.

\section{Conclusions}

The main benefit of the DACCORD concept lies in creating face-to-face communication through tangible interaction and in providing a modality to give or receive feedback. The paramedics valued that the concept would urge them to go through every step. Whereas the nurses valued that, the concept forces the communication partners to face each other at one location. Such a system would stimulate cooperation between the pre-hospital and hospital patient treatment by lending information accessibility and transparency. This newly created information chain would result in a faster and quality enhanced patient treatment and prevention of miscommunication and information loss which could be detrimental to the patients health.

\section{References}

1. Berger, B.: Patientenübergabe zwischen Rettungsdienst und Ambulanzpersonal aus Sicht des Rettungsdienstpersonals, XIII. Innsbrucker Notfallsymposium (2004)

2. Currie, J.: Improving the efficiency of patient handover. J. Emerg Nurse 10(3), 24-27 (2002)

3. Jenkin, A., Abelson-Mitchell, N., et al.: Patient handover: Time for a change? J. Accident \& Emergency Nursing 15(3), 141-147 (2007)

4. Jones, J.S., Dwyer, P.R., et al.: Patient transfer from nursing home to emergency department: outcomes and policy implications. J. Acad Emerg Med 4(9), 908-915 (1997)

5. Saffer, D.: Designing for interaction; creating smart applications and clever devices (2007)

6. Yong, G., Dent, A., et al.: Handover from paramedics: Observations and emergency department clinician perceptions. J. Emergency Medicine Australasia 20(2), 149-155 (2008) 